\title{
Design for Energy-Aware IP over WDM Networks with Hibernation Mode and Group-Node Techniques
}

\author{
Mohd Nazri Bin Mohd Warip ${ }^{1}$, Ivan Andonovic ${ }^{2}$, Ivan Glesk ${ }^{2}$, \\ R. Badlishah Ahmad ${ }^{1}$, Phaklen Ehkan ${ }^{1}$, Mohamed Elshaikh Elobaid Said Ahmed ${ }^{1}$, \\ Shamsul Jamel Elias ${ }^{1}$, Fazrul Faiz Zakaria ${ }^{1}$ \\ ${ }^{1}$ School of Computer and Communication Engineering, Univeriti Malaysia Perlis, \\ Pauh Putra Main Campus, \\ 02600 Arau, Perlis, Malaysia \\ \{nazriwarip, badli, phaklen, elshaikh,ffaiz\}@unimap.edu.my \\ ${ }^{2}$ Deparment of Electronic and Electrical Engineering, University of Strathclyde, 204 George \\ Street, Glasgow G1 1XW, United Kingdom \\ \{i.andonovic, i.glesk\}@strath.ac.uk
}

\begin{abstract}
The focus of the paper is an investigation and evaluation of energy efficient solutions in IP over WDM core networks using as a foundation, a hierarchy of hibernation modes implementing different degrees of node groupings and fibre links establishment that support a sleep state. It seeks to embed this groups-nodes strategy into an intelligent control plane implementing routing schemes targeting energy consumption, adaptive signalling and traffic engineering. A Group-Nodes mechanism is proposed as a function of topology and node distribution based on a fixed (or geographical) and random (or ownership) principle. The impact of the proposed technique on energy saving and network performance is assessed; results are presented and evaluated for various scenarios. Evaluation of this methodology indicates potential reduction in power consumption from $7 \%$ up to $15 \%$ at the expense of reduced network performance.
\end{abstract}

\section{Introduction}

With regards to zero carbon emission, the issues of green networks technology has become primary interest among researchers. Recent studies shows that rapid changes on the information and communication technology (ICT) devices and deployment of network infrastructures are having a critical effect on carbon footprint.

For this reason, the power consumption prediction as stated in [1], exhibit that the worldwide operation of network equipment accounts for $25 \mathrm{GW}$ (yearly average) of the total ICT consumption. Conversely, the joules/bit in telecommunication networks is decreasing with time, the joules/user keeps steadily increasing.

So far, the Smart Sleep Mode is the pinnacle of current green networks technology in which focusing on access networks, offering automated and low power design mechanisms. However, far too little attention has been paid to core networks.

In this paper, the research centre on the development of energy saving schemes that support the evolution of greener core IP over WDM (Wavelength Division 
Multiplexer) networks. The cornerstone of the adopted strategy is various schemes underpinned by the hibernation state implemented through a modification of the control plane, in particular for transparent network architectures under different scenarios. The research evaluated the impact and constraints that arise under this strategy, to provide useful insights on the viability of the approach for practical energy efficient savings.

\section{Potential Energy Conservation in Core IP over WDM Networks}

One of the most momentousness cost minimisation strategies in core IP over WDM networks (also sometimes known as IP over Optical or optical IP) [2][3] in terms of provisioning, operation and maintenance is savings in energy consumption. The major power consumption contributors of equipment in the network are:

-WDM chassis; receiving/transmitting equipment such as transponders modules, short-reach optical interface transponders.

- Optical Switching (OXC) Chassis, opaque/transparent optical transponders, Optical-Electrical-Optical (OEO) conversion.

- Core Router: electronic processing, traffic grooming and aggregation in the IP layer.

- Optical Amplifiers: predominately the Erbium Doped Fibre Amplifier (EDFAs).

-3R Regenerators: signal regeneration with re-timing, re-amplification and reshaping operations.

- Control Plane: signalling and routing algorithm modification.

\section{Network Energy Model Design}

In our approach in order to evaluate overall network power consumption and consumed energy per a data bit we used so called equivalent network energy model (see Figure 1) based on multilayer Internet Protocol / Generalized Multi-Protocol Label Switching (IP/GMPLS) over optical layers. In this model, a network carrier bandwidth of OC-192 and average energy consumption of $1019 \mathrm{~nJ}$ per bit was assumed following [4][5][6].

The parameter $\mathrm{G}_{\mathrm{n}}$ denotes a Router's dissipated power of $10 \mathrm{~kW}$ within its energy consumption of $1000 \mathrm{~nJ} / \mathrm{bit}$. $\mathrm{X}_{\mathrm{n}}$ represents Optical Cross-Connect $(\mathrm{OXC})$ dissipating $100 \mathrm{~W}$ and consumes $10 \mathrm{~nJ} / \mathrm{bit}$. $\mathrm{W}_{\mathrm{n}}$ denotes Wavelength Division Multiplexing (WDM) part of the node with dissipating power of $120 \mathrm{~W}$ and energy consumption of 12 nJ/bit. $A_{n}$ represents the consumption owing to Erbium-Doped Fibre Amplifiers (EDFA) within connection spans placed at $70 \mathrm{~km}$ intervals, the power consumption is estimated to be $1 \mathrm{~W}$ with energy of $0.1 \mathrm{~nJ} / \mathrm{bit}$. 


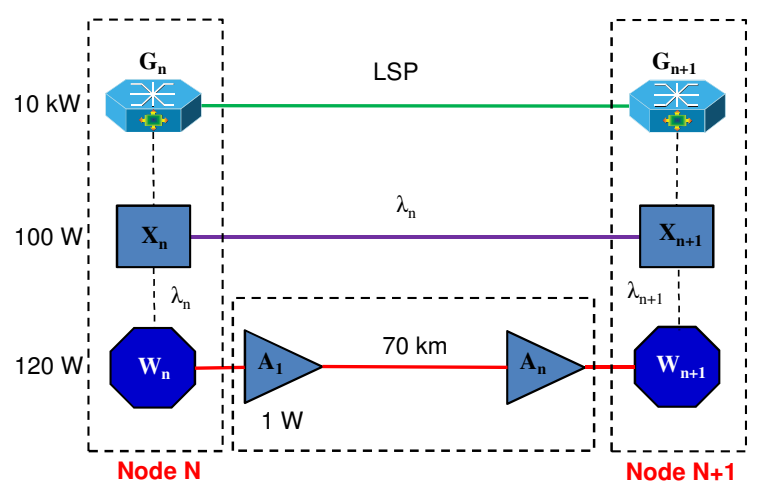

Fig. 1. An Energy Model Design for IP over WDM Networks.

\subsection{Energy per Bit}

Further we define energy per bit consumed by the node as $E_{b}=P_{T} / C$ where $P_{T}$ represents the node total power consumption and $\mathrm{C}$ is the bandwidth offered by the network link.

\subsection{Energy Consumption and Power Consumption}

The total power consumption of the link is given by:

$$
P_{\text {TOTAL }}=[\underbrace{\Delta p_{\text {CONTROL PLANE }}}_{\text {IPLAYER }}]+[\underbrace{\Delta p_{\text {OXC }}+\Delta p_{\text {WDM }}+\Delta p_{\text {TRANSPONDERS }}+\Delta p_{\text {EDFA }}}_{\text {OPTICAL LAYER }}]
$$

where $\Delta p_{\text {CONTROL PLANE }}, \Delta p_{\text {OXC }}, \Delta p_{\text {WDM }}, \Delta p_{\text {TRANSPONDERS }}$, and $\Delta p_{\text {EDFA }}$ represent power consumed by the IP/GMPLS router, OXC, WDM, transponders/transmitters, and EDFA optical amplifier, respectively.

Similarly, the total energy consumption of the link is given by:

$$
E_{\text {TOTAL }}=[\underbrace{\Delta e_{\text {CONTROL PLANE }}}_{\text {IP LAYER }}]+[\underbrace{\Delta e_{\text {OXC }}+\Delta e_{\text {WDM }}+\Delta e_{\text {TRANSPONDERS }}+\Delta e_{\text {EDFA }}}_{\text {OPTICAL LAYER }}]
$$

where $\Delta e_{\text {CONTROL PLANE }}, \Delta p_{\text {OXC }}, \Delta p_{\text {WDM }}, \Delta p_{\text {TRANSPONDERS }}$, and $\Delta p_{\text {EDFA }}$ represent power consumed by the IP/GMPLS router, OXC, WDM, transponders/transmitters, and EDFA optical amplifier, respectively.

The total energy consumption in IP over WDM networks calculated per data bit Ebit in order to support the network offered load can be defined as: 


$$
\begin{aligned}
\sum_{k=0}^{\infty} E_{\mathrm{BIT}}= & {[\underbrace{\sum_{k=0}^{n-1} \Delta p_{\text {CONTROL PLANE }}}_{\text {IP LAYER }}]+[\underbrace{\left.\frac{\sum_{k=0}^{n-1} \Delta p_{\mathrm{WDM}}}{C}+\frac{\sum_{k=0}^{n-1} \Delta p_{\text {TRANSPONDERS }}}{C}\right]+}_{\text {OPTICAL LAYER }}} \\
& {[\underbrace{\sum_{k=0}^{n-1} \Delta e_{\text {EDFA }}+(\alpha+1) \sum_{k=0}^{n-1} \Delta e_{\text {OXC }}}_{\text {OPTICAL LAYER }}]+[\underbrace{\beta}_{\text {CONSTANT }}] }
\end{aligned}
$$

where $\Delta e_{\mathrm{EDFA}}$ is the energy consumed by EDFA; $\Delta e_{\mathrm{OXC}}$ is the energy consumed by the node's OXC; $\beta$ represent the noise factor associated with the Bit Error Rate (BER) and a heat transfer rate in network equipments; and finally, $\alpha$ is number of hops.

\section{Hibernation Mode; Group Nodes Distribution Factors}

The Group Node structure in relation to the hibernation mode is illustrated in Figure 2. In this architecture, nodes consisting of IP Router and OXC are interconnected by point-to-point optical fibre links.

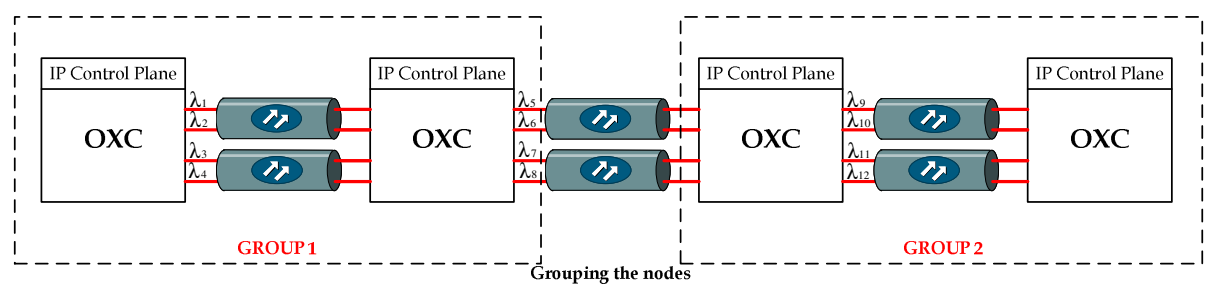

Fig. 2. Hibernation Mode: Grouped Nodes Structure.

The exchange of messages updating energy consumption profiles can be described by a sequence diagram; Figure 3 illustrates the messages sequence diagram for the transition from $\mathrm{ON}$ to OFF state. For end-to-end provisioning - from ingress to egress node - after path computation, the wavelength is reserved by signalling. Node 1 transmits a connection request along the link to reserve the wavelength and establish an end-to-end channel through the LSP Path/Resv Message. The LSP setup request is then forwarded to the next node until the message request is at the egress node. If there is an idle node at an intermediate node (in this case Node 3), after the nested hold-off timer expires, the hibernation notification message is propagated back along the path to release the reserved wavelength. The loopback LSP Resv_Confirm message will be transmitted back along the link until at the ingress node to request the suspension of the idle node. If Node 3 receives a LSP setup request message to place the node in Sleep state, it sends a Resv_Err message to acknowledge the ingress node that Node 3 is in powering off state. As a result, the network updates the routing table 
and TED topology. The ingress node releases a LSP by propagating Path_Tear message and powers down the connection to the idle node.

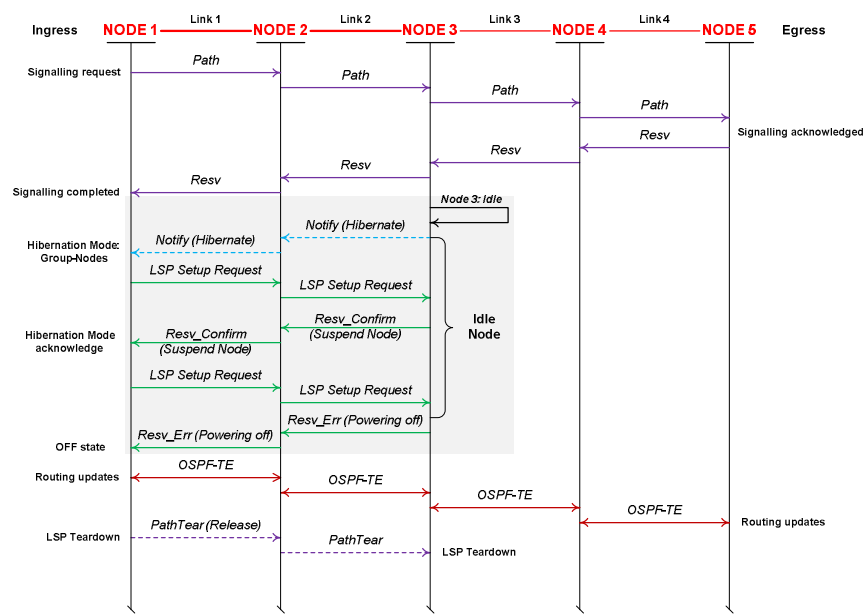

Fig. 3. Messages Sequence Diagram for transition from ON state to OFF state of Hibernation: Group-Nodes.

Figure 4 presents a message sequence diagram for the transition from OFF state to ON state. Node 3 detects traffic and changes its state to BUSY (active transition state) and full power operation is resumed, confirmed by sending a notify message to inform the adjacent node that it is in the process of waking-up. A Resv_Tear message is sent to the ingress node to notify that node is powering to ON state.

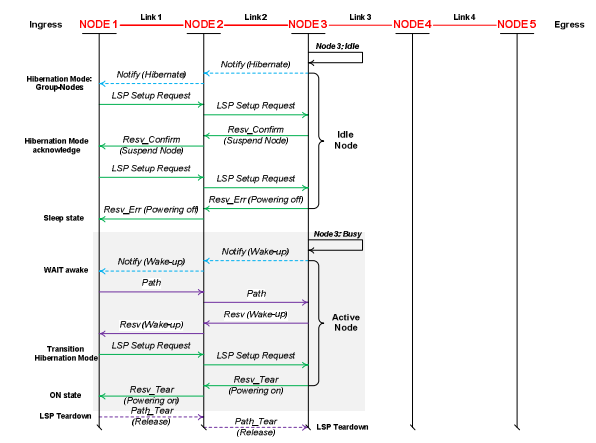

Fig. 4. Messages Sequence Diagram for transition from OFF state to ON state of Hibernation: Group-Nodes.

\subsection{Fixed (Geographical) Nodes Effect}

Fixed node or Geographical Node groups are defined as a grouping topology that contains selected neighbouring nodes and grouped as disjoint clusters. 


\subsection{Random (Ownership) Nodes Effect}

Random node or Ownership-based node groupings are defined as nodes belonging to the same owner (service provider) e.g. organization that having many entities under the same company name.

\section{Simulation Results}

The Group-Nodes hibernation mode that invokes cluster based architectures was evaluated and investigated. By dividing the nodes into several disjoint sets, as well as providing each node with geographical and ownership topology settings, produces clusters adopting sleep cycles to reduce power consumption. Figure 5 illustrates the network topology which is being utilised by the European Optical Network (EON network). The EON network has a Full Mesh Network Topology with 9 nodes and 20 bidirectional fibre links [7], [8]. The IP/GMPLS nodes are linked by bidirectional pairs of single mode fibres. The EON network topology was used in our simulations and was based on a discrete event modelling tool known as OMNet++ (Object Modular Network Tested in $\mathrm{C}++$ ). It has been assumed that all links are equal in terms of number of wavelengths (eight), that the message length is fixed at 256 bytes, and a nodal processing delay is $20 \mathrm{~ms}$. All EON network Nodes are capable to maintain information on their total power consumption as well as energy per bit consumed. Wherein, the standard GMPLS signalling and routing protocols are implemented following the Internet Engineering Task Force (IETF) standard [9][10].

The performance metrics takes into account the average power consumption, blocking probability and average request blocking [9-13]. We also assumed that lightpath requests are uniformly distributed. Note that, the inter-arrival connection requests are independent Poisson processes with an arrival rate of $\alpha$ and the queue lengths exponentially distributed with the expected service rate time of $1 / \mu$ measured in seconds. Therefore, the network offered load is $\alpha / \mu$.

Full Mesh Network Topology: European Optical Network (EON) (Figure 5). The average power dissipation and energy consumption values assigned to each node are captured in the network energy model. In this architecture, the power consumption of nodes comprises the core router $(10 \mathrm{~kW})$, OXC (100W), WDM (120W) and EDFAs (1W) placed at $70 \mathrm{~km}$ intervals along links. For example, the power consumption (reference value) between Node A and Node G (Figure 5) linked by the single mode optical fibre across a distance of $2090 \mathrm{~km}$ at a data rate of $10 \mathrm{~Gb} / \mathrm{s}$ is $29 \mathrm{~W}$. Therefore, the total power consumption between Node A and Node G is $10.249 \mathrm{~kW}$ and the energy per bit is 1024.9nJ (Equations 1-3). 


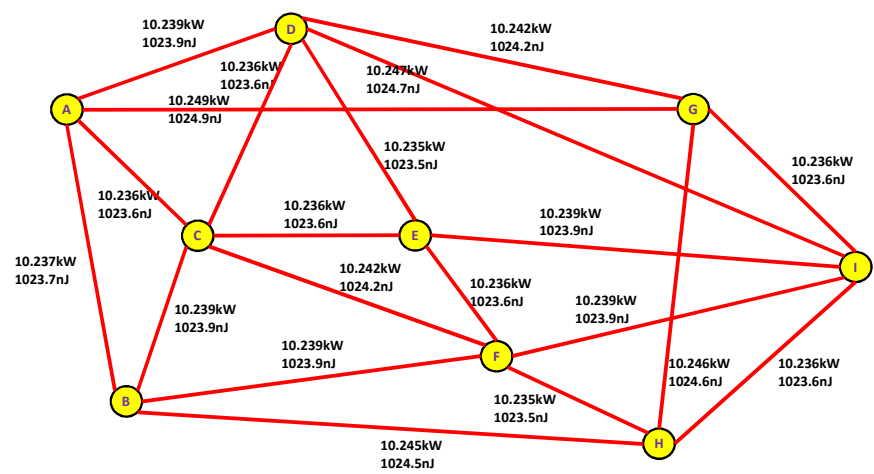

Fig. 5. Power consumption for Full Mesh European Optical Network (EON).

The proposed Hibernation concept was verified on described EON by implementing group-nodes schemes. These nodes/links are then put into "hibernation" or an "SLEEP state", in which nodes have suspended their unused functionalities (e.g. unused ports / interfaces, Mux/DeMux capabilities, signalling gates, unused wavelengths, etc.) and keep only the minimum network operation activities.

The aim is to evaluate the impact of a route to energy saving through hibernation in core optical IP network by performing the Fixed (Geographical) grouping and Random (Ownership) grouping for full mesh network topologies. Note that, these results are based on cross-layer optical/IP domains integration and previous research produced results based in the optical domain only. Therefore, results are difficult to compare in terms of power savings and network performance.

Using the full mesh EON (Figure 5), hibernation settings are applied to network nodes based on the group membership type (see node grouping categorization in Table 1).

Table 1. EON Node Grouping categorization.

\begin{tabular}{|c|l|l|}
\hline GROUP & GEOGRAPHICAL & OWNERSHIP-BASED \\
\hline G1 & Nodes $\{$ A,B $\}$ & Nodes $\{$ B,H $\}$ \\
\hline G2 & Nodes $\{$ C,D,E $\}$ & Nodes $\{$ D,E,G $\}$ \\
\hline G3 & Nodes $\{\mathrm{F}, \mathrm{G}, \mathrm{H}, \mathrm{I}\}$ & Nodes $\{$ A,C,F,I $\}$ \\
\hline
\end{tabular}

Figures 6 and Figure 7 present the average power consumption and average request blocking for various "Geographical" (adjacent nodes) groupings as a function of offered network load for the EON network mesh topology. For "All Groups HM" (case when nodes' unused functionalities are suspended) $\sim 0.137 \mathrm{~kW}$ of power is saved per node' (Figure 6) but the probability of blocking is $~ 55 \%$ (Figure 7). For Groups "G2 and G3 = HM" or Groups "G1 and G2 = HM", the power savings of $0.12 \mathrm{~kW}$ or $0.10 \mathrm{~kW}$ is obtained respectively (Figure 6) with a corresponding blocking probability of $33 \%$ or $10 \%$, respectively (Figure 7 ). 


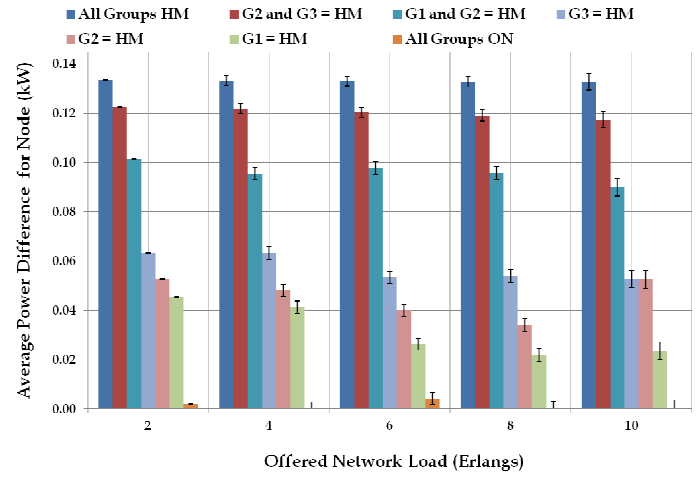

Fig. 6. Simulation results for different EON Fixed (Geographical) Node groupings. 'HM' stands for Hibernation Mode.

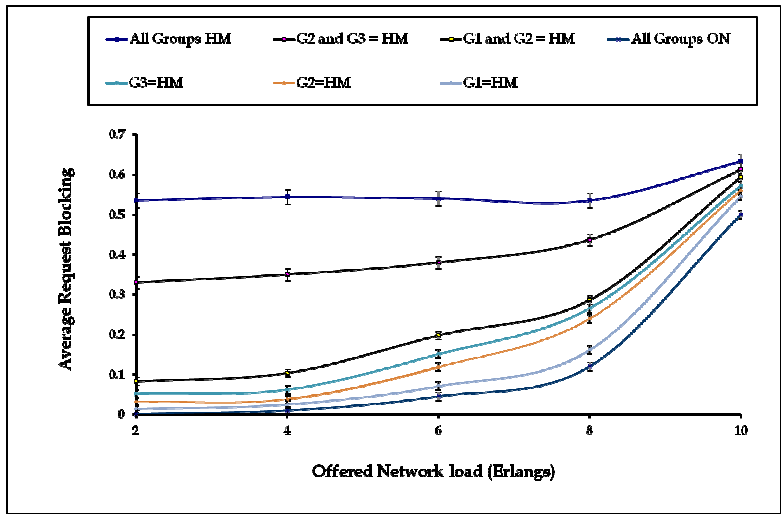

Fig. 7. Request blocking for EON Fixed (Geographical) grouping of nodes.

"All Groups ON" yields the lowest blocking probability but the power savings per node are minimal. The EON network also becomes congested for network loads exceeding 8 Erlangs. The trade-off between a reduction in energy consumption and the probability of blocking is evident.

Figure 8 depicts the average power consumption for different groups with respect to offered network load and Figure 9 presents the blocking probability for the EON ownership (random) grouping of nodes. As expected, savings in power with ownership grouping in the full mesh (EON) topology improves when compared to the partial mesh (NSFnet) topology, particularly in the case of ownership grouping. In this case, the power savings for grouping "G2 and G3" or "G1 and G2" are most significant, the improvement being $\sim 0.135 \mathrm{~kW}$ and $\sim 0.11 \mathrm{~kW}$ respectively (Figure 8 ); the corresponding blocking probability is $29 \%$ and $8 \%$, respectively (Figure 9 ). The reason for this is that the optical bypass at intermediate nodes reduces the number of required core router electrical ports in the IP layer and thereby, the energy owing to this electrical equipment is saved. The EON ownership-based node groupings (Figure 
6 and Figure 8), "All Groups = HM" and "All Groups = ON" deliver similar results as the geographical node groupings.

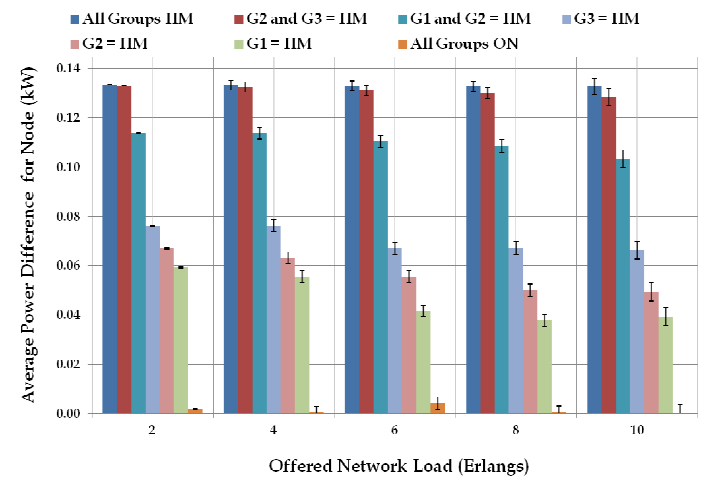

Fig. 8. Average power consumption difference per node as a function of network load for different EON random (Ownership) node grouping.

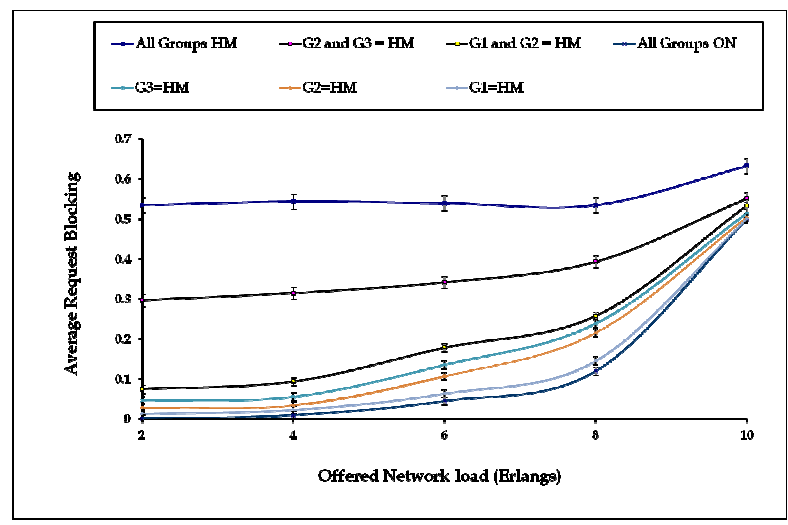

Fig. 9. Request blocking probability as a function of network load for EON random (Ownership) grouping of nodes.

\section{Conclusion}

In this paper we have presented an evaluation of an approach to energy saving in IP over WDM networks-based GMPLS control plane based on hibernation. The impact of the grouping of nodes following the principles of hibernation on network performance as a function of energy saving was examined and quantified for two representative network topologies. The approach was implemented through two grouping network node strategies - Geographical and Ownership-based. Results show that hibernation has the potential to deliver energy savings at the expense of reduced 
network performance. Results show that the "Ownership-based node groupings for "All Groups = HM" (hibernate) and "All Groups = ON" delivers similar performance as the Geographical nodes groupings.

Acknowledgments. The research leading to these results has received funding from the Ministry of Education Malaysia under grant Fundamental Research Grant Scheme (FRGS).

\section{References}

1. Pickavet, M., Vereecken, W., Dameyer, S., Audenaert, P., Vermeulen, B., Develder, Colle, D., Dhoedt, B., Demeester, P.: World Energy Needs for ICT: The Rise of Power-Aware Networking. In Proc. Advanced Networks and Telecommunication Systems, Bombay, India, (2008)

2. Musumecci, F., Tornatore, M., Pattavina, A.: A Power Consumption Analysis for IP-OverWDM Core Network Architecture. IEEE/OSA J. Optical Comms. and Network. 4 (2012) $108-117$

3. Rajagopalan, B., Luciani, J.V., Awduche D.O.: IP over Optical Networks: A Framework. RFC3717. IETF. Mar (2004)

4. Balinga, J., Ayre, R., Hinton, K., Sorin, W. V., Tucker, R. S.: Energy Consumption in Optical IP Networks. IEEE J. Lightwave Technol. 2713 (2009) 2391-2403

5. Bathula, B.G., Alresheedi, M., Elmirghani J.M.H: Energy Efficient Architectures for Optical Networks. Proc. London Communications Symposium. University College London. (2009) $1-4$

6. Cisco Systems Data Sheets. [Online]. Available: http://www.cisco.com (2013)

7. Shen, G., Tucker, R.S.: Energy-Minimized Design for IP Over WDM Networks. IEEE/OSA J. Optical Comms. and Network. 11 (2009) 176-186

8. Kim, Y., Lee, C., Kevin Rhee, J.K., Lee, S.: IP over WDM Cross-Layer Design for Green Optical Networking with Energy Proportionally Consideration. IEEE J. Lightwave Technol. 3013 (2012) 2088-2096

9. Albarrak, S.: Failure recovery in distributed GMPLS-based ip-over-optical networks. Ph.D. Thesis, Dept. Electronic \& Elect. Eng., Univ. of Strathclyde, Glasgow, UK, (2008).

10. Chabarek, J., Sommers, J., Barford, P., Estan, C., Tsiang, D., Wright, S.: Power Awareness in Network Design and Routing. Proc. 27th Conf. on Computer Communications INFOCOM. Phoenix (2008) 1130-1138

11. Ben Yoo S.: Energy Efficiency in the Future Internet: The Role of Optical Packet Switching and Optical-Label Switching. IEEE J. Sel. Quantum Electr. 172 (2011) 406-418

12. Berger, L.: Generalized Multi-Protocol Label Switching (GMPLS) signalling functional description. RFC3471. IETF. (2003)

13. Lang, J.: Generalized Multi-Protocol Label Switching (GMPLS) signalling Resource ReserVation Protocol-Traffic Engineering (RSVP-TE) Extension. RFC3473. IETF. (2003) 